\title{
Thematic issue on contribution of Artificial Intelligence to Ambient Intelligence
}

\author{
Anind K. Dey ${ }^{\mathrm{a}, *}$, Achilles Kameas ${ }^{\mathrm{b}}$ and Carlos Ramos ${ }^{\mathrm{c}}$ \\ ${ }^{a}$ Carnegie-Mellon University, USA \\ ${ }^{\mathrm{b}}$ Hellenic Open University \& Computer Technology Institute, Patras, Greece, E-mail: kameas@cti.gr \\ ${ }^{\mathrm{c}}$ Institute of Engineering - Polytechnic of Porto, Portugal, E-mail: csr@dei.isep.ipp.pt
}

Since its inception, the vision of Ambient Intelligence (AmI) holds great promise in permeating everyday life thereby changing the nature of almost every human activity [6]. In the AmI paradigm, intelligent computation will be invisibly embedded into our everyday environments through a pervasive transparent infrastructure (consisting of a multitude of sensors, actuators, processors and networks), which is capable of recognizing, responding and adapting to people and activity in a seamless and unobtrusive way. AmI promises to advance the quality and trustworthiness of a huge number of applications in all domains of human activity, including, for example, health care, education, entertainment, administration, transportation, construction, and the use of energy resources [1].

Early research efforts concentrated on the conception of innovative scenarios using the AmI vision and the development of the system architectures, software and technology required to realize them. Early architectures adopted either a bottom-up approach, leading to the development of systems consisting of networked objects having digitally augmented functionality, or a middle-out approach, focusing on the design of middleware and the development of services on top. Nevertheless, during these early years, important problems, such as heterogeneity, discovery, transparency, context representation and adaptation were encountered, but the restricted scope of the systems required solutions specific to particular settings
[3,8,12,13,19]. Hence, although Artificial Intelligence techniques were adopted, they were employed to solve specific problems within a well defined context $[4,5,16]$; some of these early architectures have found their way in commercially available components for smart homes or communicating information devices (mobile phones, personalized medical devices, media capture and processing devices, etc.).

Nowadays, a new generation of AmI technology is gradually becoming available, as large scale complex architectures and systems are being developed to support an ever increasing number of domains of human activity. Such systems are often described as Ambient Ecologies, offering a rich set of components and interfaces, having a dynamic and adaptive structure and implying that people, as well as other goalpursuing actors (agents, avatars, robots, etc.) are regarded as being their integral part [9]. Complexity results from the large number of active nodes, services and interactions, the vast number of data and information, the multitude of locations and the increasing number of users and tasks that have to be supported. In addition, as people become familiar with the AmI paradigm, human-centred requirements become more important; these include adaptation, consistency, trustworthiness, security, efficiency, multimodal interaction and controlled transparency. In addition, an important body of research is dedicated to the design of biologically inspired AmI systems, capable of exhibiting cognitive abilities, such

\footnotetext{
* Corresponding author. E-mail: anind@cs.cmu.edu.
} 
as autonomic operation, learning, intention recognition and emotions.

All these approaches (have to) rely heavily on AI to achieve their goals: AmI can't be achieved without AI [14]. Knowledge representation and management and knowledge-based reasoning have become core concerns in the new AmI systems; various AI techniques are used, including semantic models, logics, knowledge bases, ontologies and inference systems. Agent-based architectures are used to support situation recognition, pro-activity, navigation and adaptation. Computational intelligence techniques are employed to provide context representation, interpretation, planning, reasoning and intention-goal formation. Symbolic approaches, such as fuzzy logic, as well as sub-symbolic approaches, such as artificial neural networks, are used to deal with uncertainty and noise. Adaptation and evolution are supported with genetic algorithms, machine and statistical learning. Heterogeneity is dealt with the application of ontology matching and merging techniques $[2,7,10$, $11,15,17,18]$.

In this thematic issue, we have focused on the contribution of AI techniques in the deployment of AmI systems in different domains. Following the published call, we received 29 contributions, from which, after a strict peer-review procedure, we selected five to include in this third issue of JAISE. The papers constitute interesting case studies of applying AI techniques to the design of AmI systems, and we briefly introduce them here.

The paper by Ishii and Imai describes COSPI, a system that enables communication robots to interface with different sensor configurations. The authors propose the use of a set of recognition types as an abstraction layer between the sensors in the environment and the robot; these are maintained by the COSPI server, which mediates between the robot(s) and the environment. This approach enables a robot to realize a specific task in different settings, as well as to realize multiple tasks in the same environment, using the sensors it contains.

On a similar topic (that of situation detection), but following a different approach (based on semantically rich representation), the paper by Kadouche et al. relies on the interaction between the human body and its environment (defined by the authors as $\mathrm{Hu}-$ man Environment Interaction), in order to personalize and compensate for human limitations, based on the relationship between the environment and the impairment. The authors have formalized, using an implementation-independent semantic matching framework (SMF), the relationship between the user's physical characteristics and the technical parameters of the environment, so that detection of user's impairments in any given environment is possible.

The paper by Springer and Turhan presents a formal approach for modeling situation awareness, which uses Description Logic as the knowledge representation formalism to reason about the type of complex situations, by integrating context information into the situation model. The authors provide an example to justify that the use of a standard ontology language and off-the shelf reasoning tools can lead to significant reduction in the development overhead.

Kritsotakis et al. present C-NGINE, a flexible navigation guide for indoor environments that uses a combination of ontologies and rule-based proactive and reactive reasoning. The system integrates user modeling, contextual path finding and user tracking components, all using independent, tailor-made ontologies, in order to deduce the optimal path according to the user's individual preferences; in case user preferences or structure of the environment change, the system re-calculates the navigation path. The authors present a complete knowledge-based solution that expands navigation systems with capabilities exploiting the Ambient Intelligence.

The last paper researches on a topic that we expect to gain in importance in the coming years. Van den Broek et al. explore the combination of speech and electrocardiogram (ECG) signals in order to deduce people's experienced valence and arousal. The combination of these two signals has not been explored in the past, mainly because physiological measures are often obtrusive and, hence, disregarded for usercentered applications, while speech is very noise sensitive. So, the presented controlled study is one of the first showing that the combination of these two measures provides a reliable, robust, and unobtrusive method to determine a user's emotional state.

The five papers collected in this thematic issue highlight different aspects of AI contribution to AmI systems, but adopt a common set of principles: disassociation of the representations maintained by the various system components and the environment, semantically rich modeling of properties, states, situations, plans and actions, pro-active system operation based on perception, planning and reasoning, holistic approach to system design and support for multi-user, multi-task applications. We expect these principles to guide the development of the new generation of AmI systems; this can only be possible with the contribution of AI. 


\section{References}

[1] E. Aarts, Ambient Intelligence: Vision of our future, IEEE multimedia 11(1), 12-19, 2004.

[2] H. Aghajan, J.C. Augusto, C. Wu, P. McCullagh, and J.A. Walkden. Distributed Vision-based Accident Management for Assisted Living, in Proc. of the 5th International Conference On Smart Homes and Health Telematics (ICOST 2007), 196-205, 2007.

[3] C. Becker, G. Schiele, H. Gubbels, and K. Rothermel. BASE - A Micro-broker-based Middleware For Pervasive Computing, in Proc. of the 1st IEEE Inter. Conf. on Pervasive Computing and Communications (PerCom03), 443-451, 2003.

[4] E. Christopoulou, and A. Kameas. GAS Ontology: an ontology for collaboration among ubiquitous computing devices, International Journal of Human-Computer Studies (special issue on Protégé), Academic Press, 62(5), 664-685, 2005.

[5] A.K. Dey, D. Salber, and G.D. Abowd. A conceptual framework and a toolkit for supporting the rapid prototyping of context-aware applications. Human-Computer Interaction Journal, 16(2-4), 97-166, 2001.

[6] K. Ducatel, M. Bogdanowicz, F. Scapolo, J. Leijten, and J.-C. Burgelman. Scenarios for Ambient Intelligence in 2010, Tech. report, European Commission Information Society Technologies Advisory Group (ISTAG), February 2001.

[7] J. Euzenat, A. Mocan, and F. Scharffe. Ontology Alignment: An ontology management perspective. In M. Hepp, P. De Leenher, A. de Moor, Y. Sure (Eds): Ontology Management: Semantic Web, Semantic Web Services, and Business Applications, 177-206, 2007.

[8] D. Garlan, D.P. Siewiorek, A. Smailagic, and P. Steenkiste. Project Aura: Toward Distraction-Free Pervasive Computing, IEEE Pervasive Computing, 1(2), 22-31, 2002.

[9] C. Goumopoulos, and A. Kameas. Ambient Ecologies in Smart Homes, The Computer Journal, 2008; doi: 10.1093/comjnl/bxn042.

[10] H. Hagras, F. Doctor, V. Callaghan, and A. Lopez. An incremental adaptive life long learning approach for type-2 fuzzy embedded agents in ambient intelligent environments. IEEE Transactions on Fuzzy Systems, 15(1), 41-55, 2007.

[11] T. Heinroth, A. Kameas, C. Wagner, and Y. Bellik. Realizing Adaptive Ambient Ecologies through an ontologybased Multi-Agent System, in Proc. of the 2009 IEEE International Symposium on Intelligent Agents, 2009.

[12] L.E. Holmquist, H.-W. Gellersen, A. Schmidt, M. Stro-hbach, G. Kortuem, S. Antifakos, F. Michahelles, B. Schiele, M. Beigl, and R. Mazé. Building Intelligent Environments with Smart-Its". IEEE Computer Graphics \& Applications, 24(1), 56-64, 2004.

[13] S. Lalis, A. Savidis, A. Karypidis, J. Gutknecht, and C. Stephanides. Towards Dynamic and Cooperative MultiDevice Personal Computing. in: Streitz, N., Kameas, A., Mavrommati, I. (eds.) The Disappearing Computer, LNCS, Springer, Heidelberg, vol. 4500, 182-204, 2007.

[14] C. Ramos, J.C. Augusto, and D. Shapiro. Ambient intelligence - the next step for artificial intelligence. IEEE Intelligent Systems, 23(2), 15-18, 2008.

[15] A. Ranganathan, C. Shankar, and R. Campbell. Application Polymorphism for Autonomic Ubiquitous Computing, in Proc. of the 1st Annual International Conference on Mobile and Ubiquitous Systems, 402-411, 2004.

[16] M. Roman, C. Hess, R. Cerqueira, A. Ranganathan, R. Campbell, and K. Nahrst. Gaia: A Middleware Infrastructure to Enable Active Spaces. IEEE Pervasive Computing, 1(4), 74-83, 2002.

[17] C. Rousseau, Y. Bellik, F. Vernier, and D. Bazalgette. A framework for the intelligent multimodal presentation of information. Signal Processing, 86(12), 3696-3713, 2006.

[18] B. Schattenberg and S. Biundo. A unifying framework for hybrid planning and scheduling, in Proc. 29th German Conference on Artificial Intelligence (KI 2006), 361-373, 2006.

[19] N.A. Streitz, C. Rocker, T. Prante, D. v. Alphen, R. Stenzel, and C. Magerkurth. Designing Smart Artifacts for Smart Environments, IEEE Computer 38(3), 41-49, 2005. 